\title{
References
}

Ackoff, R. (1960). Systems, Organizations and Interdisciplinary Research, General Systems Yearbook.

Ackoff, R. (1970). A Concept of Corporate Planning, John Wiley, New York.

Ackoff, R. (1971). 'Towards a System of Systems Concepts', Management Science, Vol. 17, No. 11.

Ackoff, R. (1972). 'The Systems Revolution', Long Range Planning, 7, 2-20.

Ackoff, R. (1981). Creating the Corporate Future, John Wiley, New York. Alley, B. \& Bender, M. (1998). Greenland Ice Cores - Frozen in Time, Scientific American, February 1998.

Anderson, W. (1987). To Govern Evolution, Harcourt, New York.

Ashby, R. (1964). An Introduction to Cybernetics, Chapman \& Hall, London.

Asimov, I. (1968). I Robot, Grafton Books, London.

Aulin, A. \& Ahmavaara, A. (1979). 'The Law of Requisite Hierarchy', Kybernetes, No. 8.

Austin, J. (1978). Chance and Creativity: The Lucky Art of Novelty, Columbia UP, New York.

Bateson, G. (1972). Steps to an Ecology of Mind, Ballantine, New York. Beer, S. (1972). Brain of the Firm, Penguin Press, London.

Beer, S. (1979). The Heart of Enterprise, John Wiley, New York.

Bergström, M. (1990). Hjärnans Resurser, Seminarium Förlag AB, Jönköping.

von Bertalanffy, L. (1955). 'General Systems Theory', Main Currents in Modern Thought, Vol. 71, No. 75.

von Bertalanffy, L. (1967). Robots, Men and Minds, Publisher, New York.

Bidgoli, H. (1989). Decision Support Systems, McGraw-Hill, New York. Bolonkin, A. (1999). 'The Twenty-first Century: The Advent of the Nonbiological Civilization and the Future of the Human Race', Kybernetes, Vol. 28, No. 3. 
Boulding, K. (1956). 'General Systems Theory - The Skeleton of Science', Management Science, No. 2.

Boulding, K. (1964). 'General Systems as a Point of View' in A. Mesarovic's, Views on General Systems Theory, John Wiley, New York.

Boulding, K. (1973). A Ballad of Ecological Awareness. The War Industry, Transaction Books, New Brunswick, New Jersey.

Boulding, K. (1978). Ecodynamics, Sage Publications, London.

Boulding, K. (1985). The World as a Total System, Sage Publications, London.

Bowler, D. (1981). General Systems Thinking, North Holland, New York. Burns, J. (1977). 'Converting Signed Digraphs to Forrester Schematics', IEEE, Vol. SMC-7, No. 10.

Campbell, N. (1953). What is Science? Dover Publications, New York. Catton, W. (1982). Overshoot, University of Illinois Press, London. Checkland, P. (1981). Systems Thinking, Systems Practice, John Wiley \& Sons, Chichester.

Cherry, C. (1966). On Human Communication, MIT Press, London. Churchman, W. (1979). The Design of Inquiring Systems: Basic Concepts of Systems and Organizations, Basic Books, New York.

Cook, N. (1980). Stability and Flexibility. An Analysis of Natural Systems, Pergamon Press, New York.

Coram, R. (2002). Boyd: The Fighter Pilot Who Changed the Art of War, Little Brown \& Company, ISBN 0316881465.

Davis, S. \& Lawrence, P. (1977). Matrix, Addison-Wesley, Reading, Massachusetts.

Dawkins, R. (1989). The Selfish Gene, Oxford University Press, Oxford. Deutsch, K. (1966). The Nerves of Government, The Free Press, New York.

Dörner, D. (1980). 'On the Problems People Have in Dealing with Complexity', Simulation and Games, No. 1.

Dreyfus, H. \& Dreyfus, S. (1986). Mind Over Machine, Basil Blackwell, New York.

Edelman, G. (1991) in M. Benedikt's, Cyberspace, MIT Press, Cambridge, Massachusetts. 
Einstein, A. (1921). Geometrie und Erfahrung, Sitzungsberichte der Preussichen Akademie der Wissenschaft Verlag der Akademie der Wissenschaften, Berlin.

Evans, C. (1980). The Micro Millennium, Viking Press, New York. Fivaz, R. (1989). L'ordre et la volupté, Presses Polytechniques Romandes, Lausanne.

Flood, R. \& Carson, E. (1988). Dealing with Complexity, Plenum Press, New York.

Flood, R. \& Jackson, M. (1991). Creative Problem Solving, John Wiley, New York.

Flood, R. \& Jackson, M. (1991). Critical Systems Thinking: Directed Readings, John Wiley, Chichester.

Flood, R. \& Romm, N. (1996). Critical Systems Thinking: Current Research and Practice, Plenum, New York.

Forrester, J.W. (1969). Urban Dynamics, MIT Press, Cambridge, Massachusetts.

Forrester, J.W. (1971). World Dynamics, Wright Allen, Cambridge, Massachusetts.

Freud, S. (1961). The Interpretation of Dreams, Wiley, New York.

Fuller, B. (1970). Operating Manual for Spaceship Earth, Carbondale III, Southern Ill. University Press, New York.

Fuller, B. (1992). Cosmography, Macmillan, New York.

van Gigch, J. (1978). Applied General Systems Theory, 2nd edition, Harper \& Row, New York.

van Gigch, J. (1991). System Design Modeling and Meta-modeling, Plenum, New York.

von Glaserfeld, E. (1987). 'The Construction of Knowledge: Contributions to Conceptual Semantics' Intersystems Seaside, California.

Greiner, L. (1972). Evolution and Revolution as Organizations Grow, Harvard Business Review, July-August.

Hillis, D. (1985). The Connection Machine, MIT Press, Cambridge, Massachusetts.

Hitchins, D. (1992). Putting Systems to Work, John Wiley, New York. Holling, C. (1977). New Directions in Futures Research, eds. H. Linstone and A. Simmonds, Addison-Wesley, Reading, Massachusetts. 
Janis, I.L. (1972). Victims of Groupthink, Houghton Mifflin, Boston. Jantsch, E. (1980). The Self-Organizing Universe, Pergamon New York. Jaques, E. (1976). A General Theory of Bureaucracy, Ashgate, Burlington, USA.

de Jardin, T. (1947). The Phenomenon of Man, Harper \& Row, New York.

Jaynes, J. (1982). The Origin of Consciousness in the Breakdown of the Bicameral Mind, Houghton Mifflin, Boston, Massachusetts.

Jordan, J. (1968). Themes in Speculative Psychology, Tavistock Publications, London.

Katz, D. \& Kahn, L. (1966). The Social Psychology of Organizations, John Wiley, London.

Kelly, K. (1994). Out of Control, Addison Wesley, New York.

Kerr, M. \& Bowen, M. (1988). Family Evaluation, W.W. Norton \& Company, New York.

Kirchner, J.W. (1991). The Gaia Hypothesis: Are They Testable? Are They Useful? in S. Schneider's, Scientists on Gaia, Cambridge, MIT Press, New York.

Klir, G. (1985). Architecture of Systems Problem-Solving, Plenum, New York.

Klir, G. (1991). Facets of Systems Science, Plenum, New York.

Koestler, A. (1967). The Ghost in the Machine, Arkana, London.

Kroenke, D. (1989). Management Information Systems, McGraw-Hill, New York.

Langefors, B. (1993). Theoretical Analysis of Information Systems, Studentlitt, Lund.

Langton, C. (1989). Artificial Life, Santa Fé Institute Studies in the Science of Complexity, Vol. 6, Addison-Wesley, Redwood City. Laszlo, E. (1972). Introduction to Systems Philosophy, Harper \& Row, New York.

Laszlo, E. (1972). The World System, George Braziller Inc., New York. Libet, B. (1985). 'Unconscious Cerebral Initiative and the Role of Conscious Will in Voluntary Action', Behavioral and Brain Sciences, No. 8.

Lilienfeld, R. (1978). The Rise of Systems Theory, John Wiley, New York. 
Litterer, J. (1969). Organizations: Systems, Control and Adaption, John Wiley, New York.

Lovelock, J. (1988). The Ages of Gaia, Norton \& Co., New York (reissued OUP, Oxford 1995).

Lovelock, J. (1979). Gaia: A New Look at Life on Earth, Oxford University Press, Oxford (reissued 1995).

MacKay, D. (1969). Information, Mechanism and Meaning, MIT Press, London.

MacLean, P. (1973). A Triune Concept of the Brain and Behavior, University of Toronto Press, Toronto.

Maslow, A. (1954). Motivation and Personality, Harper Collins, New York.

Mason, R. \& Mitroff, I. (1981). Challenging Strategic Planning Assumptions, John Wiley, New York.

Maturana, H. \& Varela, V. (1992). The Tree of Knowledge, Shambala, London.

Miller, J. (1956). The Magical Number Seven, Plus or Minus Two, Psychology Review, 63(2), 81-97.

Miller, J. (1978). Living Systems, McGraw-Hill, New York.

Miller, J. (1990). 'Introduction: The Nature of Living Systems', Behavioral Science, Vol. 35, No. 3, La Jolla, California.

Morgan, G. (1986). Images of Organization, Sage, London.

Moss, C. (1988). Elephant Memories, Legenda, New York.

Nadler, G. \& Hibino, S. (1990). Breakthrough Thinking, Prima Publishing Rocklin, California.

Namilov, V. (1981). Faces of Science, iSi Press, Philadelphia.

Nörretranders, T. (1993). Märk Världen, Bonnier Alba, Stockholm.

Payne, R. (1971). 'Song of Humpback Whales', Science, Vol. 173, August, p. 585.

Popper, K. (1959). The Logic of Scientific Discovery, Routledge \& Kegan, London.

Powers, W.T. (1973). Behavior: The Control of Perception, Aldine de Gruyter, Hawthorne, New York.

Pribram, K. (1969). 'Languages of the Brain, The Neurophysiology of Remembering', Scientific American, January. 
von Reibnitz, U. (1988). Scenario Techniques, McGraw-Hill, Hamburg. Rittel, H. \& Webber, M. (1974). 'Dilemmas in General Theory of Planning', Systems and Management Annual 1974, Petrocelli, New York.

Rucker, R. (1982). Software, Penguin Books, London.

Rumelhart, D. (1986). Parallel Distributed Processing, A Bradford Book, MIT Press, London.

Salk, J.E. (1983). Anatomy of Reality, Greenwood Publishing Group Inc., Westport, Connecticut.

Samuelson, K. (1977). General Information Systems Theory in Design, Modelling and Development, Institutional Paper, Informatics and Systems Science, Stockholm University.

Samuelson, K., Borko, H. \& Amey, G. (1977). Information Systems and Networks, North-Holland, Amsterdam.

Schleicher, A. (1888). Die Deutsche Sprache, Cotta, Stuttgart.

Schneider, S. \& Boston, P. (1993). Scientists on Gaia, MIT Press, London. Schumacher, E. (1973). Small is Beautiful, Harper \& Row, New York. Schumacher, E. (1978). A Guide for the Perplexed, Abacus, London. Shannon, C. \& Weaver, W. (1964). The Mathematical Theory of Communication, University of Illinois Press, Urbana, USA.

Simon, H. (1969). The Sciences of the Artificial, MIT Press, London. Simon, H. (1976). Administrative Behavior, The Free Press, New York. Skyttner, L. \& Fagrell, F. (1988). 'Minimizing Human, Economic and Ecological Losses at Sea' TRITA IS-5152, Stockholm University. Skyttner, L. (1993). 'The Distress Signal as a System Function', Kybernetes, Vol. 22, No. 3.

Smuts, J. (1973). Holism and Evolution (reprint), Greenwood Press, Westport, Connecticut.

Steward, J. (2003). Evolution's Arrow, Chapman Press, London. Stonier, T. (1990). Information and the Internal Structure of the Universe, Springer-Verlag, London.

Tajfel, H. \& Fraser, C. (1978). Introduction to Social Psychology, Penguin Books, Harmonsworth, United Kingdom.

Taylor, A. (1973) in E. Laszlo's, The World System, Braziller, New York. Thompson, F. (1951) in G. Cumberlege's, Poems of Francis Thompson, Oxford University Press, Oxford. 
Toffler, A. (1980). The Third Wave, Collins, London.

Turchin, V.F. (1977). The Phenomenon of Science: A Cybernetic Approach to Human Evolution, Columbia University Press.

Tustin, A. (1955). Automatic Control, Simon \& Schuster, New York.

Ulrich, W. (1983). Critical Heuristics of Social Planning, Haupt, Berne. Uris, A. (1986). 101 of the Greatest Ideas in Management, John Wiley, New York.

Waldrop, M. (1992). Complexity: The Emerging Science at the Edge of Order and Chaos, Simon \& Schuster, New York.

Warman, A. (1993). Computer Security within Organizations, Macmillan Press, London.

Watt, K. \& Craig, P. (1988). 'Surprise, Ecological Stability Theory' in C.S. Holling's, The Anatomy of Surprise, John Wiley, New York. Weawer, W. (1948). 'Science and Complexity', American Scientist, Vol. 36, No. 194.

Weinberg, G. (1975). An Introduction to General Systems Thinking, John Wiley, New York.

Wiener, N. (1948). Cybernetics or Control and Communication in the Animal and the Machine, John Wiley, New York.

Wurman, R.S. (1991). Information Anxiety, Pan Books, London. Zuchov, G. (1979). The Dancing Wu-Li Masters, Bantam Books, Toronto. 
This page is intentionally left blank 


\section{Name Index}

A

Ackoff, $\mathrm{R} \quad 41,58,72,395,396,460,492$

Adler, A 268

Ahmavaara, A 100

Alley, B 313

Andersson, $\mathrm{W} \quad 495$

Aristotle 49,140

Ashby, R 57, 100, 257

Asimov, I 350

Aulin, A 100

Austin, J 424

B

Bacon, F 486

Barrow, $\mathrm{G} 22$

Barrow, J 261

Bateson, G 234

Baudot 253

Beer, $S \quad 110,111,132,137,139,187$, 203, 257, 392

Bender, M 313

Bergström, M 286

Bertalanffy, L $\quad 39,53,56$

Bidgoli, H 406

Bohr, N 24, 29, 32, 410

Bolonkin, A $348,349,351$

Boltzmann, L 20

Boulding, $\mathrm{K} \quad 39,47,51,57,79,110$, $111,166,495$

Bowen, M 110, 192, 194, 196

Bowler, D 51, 52

Boyd, G 416

Boyle, R 114

Brahe, $\mathrm{T} 9$

Bremerman 231

Brillouin, L 22
Broca, P 266

Bruno, G 9, 11

Buddha 268

Buller, R 24

Burns, J 479

C

Caesar 278

Campbell, N 49

Carson, E $\quad 470,472$

Catton, $W \quad 487,493$

Chardin de T 110, 151-153, 155, 156, 183,268

Checkland, $\mathrm{P} \quad 110,175,481,482$

Cherry, C 215, 226

Churchman, $W \quad 52,53,57$

Clausius, $\mathrm{R} \quad 20$

Coakley, T 413

Collings, J 27

Comte, A 19, 33

Conway, $\mathrm{H} \quad 341,342,345$

Cook, N 110, 170-173, 203

Copernicus, $\mathrm{N} \quad 9$

Coram, R 416

Craig 102

Csikszentmihalyi 335

D

Damasio, A 267

Darwin, C 19, 298, 382

Davis, $S \quad 373$

Dawkins, $R \quad 263$

Descartes, $\mathrm{R} \quad 10$

Deutsch, K 87

Deway, J 390

Dörner, D 427 
Dresher 393

Dreyfus, $\mathrm{H} \quad 334$

Dreyfus, S 334

E

Edelman, G 283

Einstein, A 23-25, 27, 29, 32, 33, 98, $109,110,231,234$

Elliot, $\mathrm{T} \quad 276$

Ernst, R 417

Etzioni, A 365

Evans, C 504

\section{$F$}

Fayol, $\mathrm{H} \quad 362,363$

Fivaz, $R \quad 55,268$

Fleming 202

Flood 393

Flood, $R \quad 105,470,472,482,507$

Forrester, J 43, 475, 493

Fraser, C 214

Freud, $S \quad 32,192,269,270$

Fuller, B 31, 485, 502

G

Galenos 7

Galilei, G 9, 10, 11, 27

Gibson, W 453-455

Gigch van, J $\quad 44,54,459,462,496$

Glaserfeld von, E 57

Gödel, K 104, 105

Goete, W 50

Golding, W 140

Gore, A 449

Grainer, L 381

Gutenberg $\quad 447$

$\mathbf{H}$

Haldane, J 3

Hartley, $R \quad 250$

Hartston, W 328
Hegel, F 49

Heisenberg, W 24, 29, 109

Hibino, S 464

Hillis, D 345

Hitchins, D 58, 103

Holling, C 397

Hoos, I 499

Hutton, J 140

J

Jackson, M $\quad 105,482$

Janis, I 426

Jantsch 285

Jaques, E 110, 197, 198, 201-203

Jaynes, J 174, 270, 271

Johnsson, S 308

Jordan, J 110, 178-180

Jung, C 268

K

Kahn, L 63

Kant, I 28

Kasparov, G 328

Katz, D 63

Kelly, K 152

Kelvin, W 20

Kepler, J 9, 192

Kerr, M 192

Kirchner, J 152

Klir, G 58, 110, 162-164

Knox, R 28

Koestler, A 67

Kroenke, D 406

Ktesibios 75

Kurtzweil, R 350

L

La Mettrie 13

Langefors, B 229

Langton, C 340,343-345

Laplace, $\mathrm{P} \quad 13$ 
Laszlo, E $\quad 110,165-167$

Lawrence, $\mathrm{P} \quad 373$

Lawson, J 414, 415, 417

Libet, B 272-293

Lilienfeld, R 498, 499, 504

Linné, $K$ von 116

Linstone, $\mathrm{H} \quad 74$

Litterer, J 53

Lovelock, J 110, 140, 141, 143, 144, $147,150,257$

\section{$\mathbf{M}$}

MacKay, D 226

MacLean, P 270, 283

Margulis, L 140

Marx, K 265

Maslow, A 381, 389

Mason 482

Maturana, $\mathrm{H} \quad 60$

Maxwell, J 20, 82, 233

Mayo, E 360

Metcalfe, B 433

Miller, J 61, 100, 104, 110, 111, 118, $120,123,147,212,257,381$

Minsky, M 327

Mitroff 482

Morgan, G 387

Morris Jr, R 347

Morse, $S \quad 253,244,245$

Moss, C 216

$\mathbf{N}$

Nadler, G 464

Namilov, V 110, 189, 190, 192, 203

Newton, I 13, 18, 21, 23, 27, 32, 33, 192

Nietsche, F 428

Nörretranders, T 236

O

Ohm, G 114
$\mathbf{P}$

Paracelsus 140

Payne, R 216

Picasso, P 32

Planck, M 24, 29, 32

Plato 257

Pope, A 27, 56

Popper, K 262

Powers, W 110, 185-189

Pribram, C 289

Proust, $M \quad 32,450$

Ptolemy 6

Pythagoras 20,48

$\mathbf{R}$

Reagan 370

Rittel, $\mathrm{H} \quad 457,460$

Romm, N 507

Rothstein, J 141

Rucker, R 268

Rumelhart, D 282

S

Salk, J 110, 180-185

Samuelson, $\mathrm{K} \quad 430,434,482$

Saussure de, F 50

Scheffler, J 23

Schelling, 297

Schleicher, A 190

Schönberg 32

Schumacher, E 261, 263

Searle, J 322

Selznick, P 365

Shannon, C 207, 211, 219, 221-226, 230, 254

Simon, $\mathrm{H} \quad 357,397$

Skyttner, L 212, 475

Smith, A 353

Smuts, J 50

Spinoza 50

Steele, M 46 
Steward, J 299, 300

Stonier, T 231

Szent-Gyorgi 256

Szilard 231

\section{$T$}

\section{Tajfel, $\mathrm{H} \quad 214$}

Taylor, A 110, 156, 158

Taylor, F 358, 359

Thompson, $\mathrm{F} \quad 12$

Thompson, $W \quad 500$

Tipler, F 261

Toffler, A 432

Turchin, $V \quad 70$

Turing, A 326

Tustin 107

$\mathbf{U}$

Updike, J 26

Uris, A 367

$\mathrm{V}$

van Valen 103

Varela, V 60

Vaucanson de J 341

Vernandsky, V 153

Voltaire, $\mathrm{F} \quad 4$
W

Waldrop, M 302

Warman, A 346

Watson, L 279

Watt, J 83

Watt, K 102

Weaver, $W \quad 207,230$

Webber, M 457, 460

Weber, M 363

Weinberg, G $36,42,48,97,100$

Weinberg, S 261

Weintraub, J 327

Weiss, $P \quad 57$

Weizenbaum, J 319

Wertheimer, $\mathrm{M} \quad 50$

Wiener, $\mathrm{N} \quad 45,75$

Wilde, $\mathrm{O} 4$

Wurman, R 204

$\mathbf{Y}$

y Gasset, O 294

Yovits, $\mathrm{H} \quad 417$

$\mathbf{Z}$

Zuchov, G 37 


\section{Subject Index}

A

activator 91

ad hoc organization $\quad 374$

adaptability 70

adjustment processes

125

aggregate 58

alchemy 6

algorithm 395

algorithmic complexity

45

alienation 365

allopoietic 60

amplifier 133

analogies 39

analogue 98

analogue communication

analysis 34,42

anasynthesis 42

anti-normativism 19

anticipatory 81

antropic principle 261

archetypes 269

artefact 44

artificial life 339,340

associative memory 291

associator 124,257

astrology 6

astronomy 9,23

atmosphere 143

attenuator 134

autonomy 35

autopoiesis 60

axiom 92

B

basic control cycle 90

behaviourists 321 bifurcation $\quad 302$

big bang 27

biodiversity 149

biogenesis 154

bionics 46

biosphere 142

boundary 129

box-

black 78

gray 79

white 79

brain plasticity 282

brainstorming 423

brainwriting 424

Broca's area 266

buffering 80

bureaucracy 363

butterfly effect 74

C

C $^{3}$ I 46

capta 275

causality 14

centralization 67,369

centrifugal regulator 83

channel-

continous 250

discrete 250

channels of-

communication 223

observation 223

Chess test 328

Chinese Room 322

Clan-think 426

clepsydra 75

clockwork 14, 112 
code- 252

$$
\text { alpha } 128
$$$$
\text { beta } 128
$$

gamma 128

cognitive deficiency 426

cognitive dissonance 189,391

command \& control 413

communication 211

comparator 91

complementarity principle 24

complexification 153

complexity

algorithmic 45

computational 328,401

component 78

computational ethology $\quad 340$

computer virus $\quad 346-348$

consciousness-

\section{self 267}

omni 268

constructivism 57

contingency theory 366

control 77

control theory 185

controller 90

convergence 153

converter 123

cosmological principle 27

cosmology 28

counter-measure team 99

creationism 426

cryptography 206

cue 208

cybernetics 75

cyberpunk 455

cyberspace 454

cyclic 68

D

damping 223

Daysiworld 144 death 94
decider 91,125
decoder $\quad 124$
delay 90
demon-

Laplace's 13

Maxwell's 20, 233

descision-

matrix 402

tree 402

design 43

designer 52,91

detectability 209

detector 91

deterioration 94

determinism 14,18

deterministic chaos 74

differentiation 54

digital compression 432

discriminator 91

disorder 20

distorsion 223

distributor 123

drill down 410

dualism 10

dynamic equilibrium 93

E

Economic Man 314

effektor 91

eidetic memory 274

element 66

embodiment 324

emergence 40

emergent properties 69

emergents 127

empathy 55,266

empirical referents 51

empiricism 12, 42

encoder 125

endogenous 67 
entropy- $20,71,233$

$$
\text { information } 247
$$

maximum 247

minimum 247

relative 247

environment 63

epiphenomenon 258

equifinality 71,101

equilibrium 76

Eros 269

error tolerances $\quad 257$

Esperanto 338

evolution 94

evolutionary paradigm

exformation 236

existential levels 262

exogenous 67

expansionism 34

extruder 124

\section{F}

feedback-

extrinsic $\quad 87$

first order 86

higher order 86

intrinsic 87

negative 83

positive 84

second order 86

feedforward 81

fictionalism 57

fin de siècle 20

finalistic theory 153

flow 335

framework 112

frontesterion 392

functional organization 360,371

functionalism 320

G

Gaia $\quad 140$

game theory 391

55 gaming 42,99

General Systems Theory 40

genogram 194

gentle degradation 283

geogenesis 154

geophysiology 140

geosphere 143

gestalt psychology 50

gnome 6

goal-setter 91

greenhouse civilization $\quad \mathbf{1 4 8}$

groupthink 426

H

hard feedback 384

Hawtorne-effect 361

hemisphere 171

heterarchy 65

heteropoietic 60

heuristics 332, 395

hierarchy 54

holism 50

holistic 38

hologram 289

holon 67

homeokinesis 93

homeokinetic plateau 93

homeostasis 93

Homo Economicus 397

Homo Habilis 265

homomorphism 40

hydrosphere 143

hypothesis 96

I

idealism 28

indeterminism 21

infological equation 229

infology 206

informability 209

informatics 44,430 


$\begin{array}{lc}\text { information- } & \\ \text { descriptive } & 228 \\ \text { input overload } & 128 \\ \text { input underload } & 129 \\ \text { metrical } & 228 \\ \text { physics } & 231 \\ \text { science } & 206 \\ \text { selective } & 228 \\ \text { structural } & 228\end{array}$

system $75,135,192,206,208,235$, $323,379,387,392,417,428$

theory 205

ingestor 123

input 78

intelligence-

amplifier 319

spectrum 276

interactive mode $\quad 212$

interface 65

internal transducer 124

Internet 440

intersubjectivity 16

intrasubjectivity 16

invincible hand 353

isomorphism 39

iteration 303

$\mathrm{J}$

jamming 211

$\mathbf{L}$

laboratory 15

lag 90

law of laws 39

law-

first of thermodynamics 20

second of thermodynamics 20

third of thermodynamics 20

level

pragmatic 219

semantic 219

syntactic 219
Libido 269

linguistics 206

listening typewriter 338

localizability 209

logic bomb 347

$\mathbf{M}$

machine age 13

macrohierarchy 166

macroorganism 189

macroscope 35

man-made 59

management cybernetics 46,352

mapping 330

marker 124

matrix-design 372

meaning 205, 207, 212, 219, 230

meme 263

memory 125

mentefact 61

message-

external 210

internal 210

meta 210

messes 36

meta-

communication 209

discipline 41,497

theory 56

metabolism 60, 119

methodologies-

hard 481

soft 481

microhierarchy 166

microscope 35

mnemonics 234

model- 90

analogue 90

conceptual 91

descriptive 91

explanatory 91

iconic 90 
model-

mathematical 91

predictive 91

prescriptive 91

symbolic 90

verbal 91

modelling 43

monerans 118

monism 18

morality 265

Morse code 244, 245, 253

multifinality 54

multilateral structure 69

N

natural channels

213

negentropy 21

neocortex 285

neural nets 329

neural network 330

neurocybernetics 132

neurodynamics 287

neutrality 15

noise-

black 252

pragmatic 251

semantic 251

syntactic 251

transmission 251

white 251

nominalism 19

non-intervention 15

non-locality 26

nöogenetics 116

nōosphere 153

norm 9

O

objectivity 15

OODA-loop $\quad 416,417$

operations research 38

optimizing 395 order 51

organizational recursion 133

organized-complexity 71

organized-simplicity 72

output 78

output transducer $\quad 125$

$\mathbf{P}$

packet switching $\quad 440$

panpsychism 259

paradigm- 96

renaissance 8

scientific 18

scholastic 5

parallel processing 329

pattern recognition 338

phase transition 303

pheromones 128

physiology 186

planning loop 81

Platonic bodies 6

point of Omega 154

positivism 19

potentiality 289

praxeology- $\quad 390$

descriptive 391

normative 391

Prisoner's Dilemma 393

producer 123

program 77

prostheses 122

protistans 118

psychogenesis 147

Q

quantization 158

quantum-

mechanics 25

theory 24

$\mathbf{R}$

rationalism 12 
receptor 91

recursion 133

redesign 43

reductionism 14

redundancy-

pragmatic 249

semantic 249

syntactic 248

reference signal 188

reference standard 91

regression 129

relational universals 52

relativity 23

repeatibility 16

repetitionality 17

replication 60

representative substitutions 207

reproducer 123

requisite variety 133,135

robotics 325,339

\section{S}

salamander 6

sampling theorem 250

satellite 35

satisficing 393

schemata 283

science of complexity 44

scientific-

management 358

Method 17

paradigm 18

revolution 9

scientism 22

self-organization 294

self-reference 137

self-transcendence 325

semiotics 206

sensor 83

sensory deprivation

servo 83

sexual system 116 sign $\quad 207$

signal 208

signalling-

deceptive 210

false 210

supplementary 210

simulation 41

single-directed mode 212

singularity 27

soft feedback 384

somnambulism 270

space-

abstract 64

cognitive 64

existential $\quad 64$

perceptual 64

pragmatic 64

spectral effect 25

speech recognition 336

stability 70

standard model 27

static properties 34

steady-state 76

stenography 252

structuralism 50

structure 57,69

structure-functionalism 365

sublimation 269

superbrain 351

superdeterminism 14

Superego 269

superorganism 379

supporter 124

sylf 6

symbiosis 143

symbol 208

synaesthesia 281

synectics 424

synergetic 69

synthesis 34

syntropic 31 


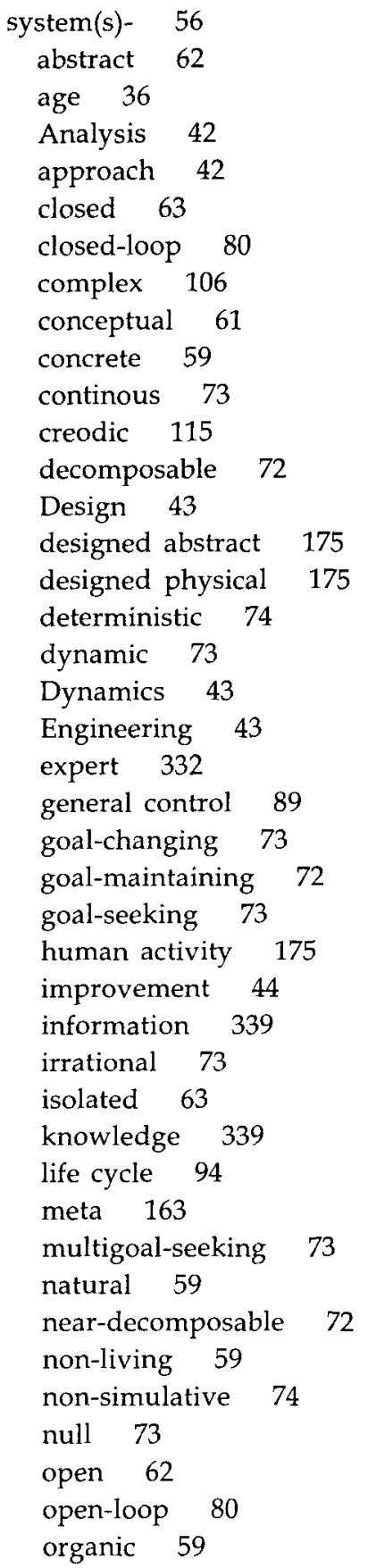

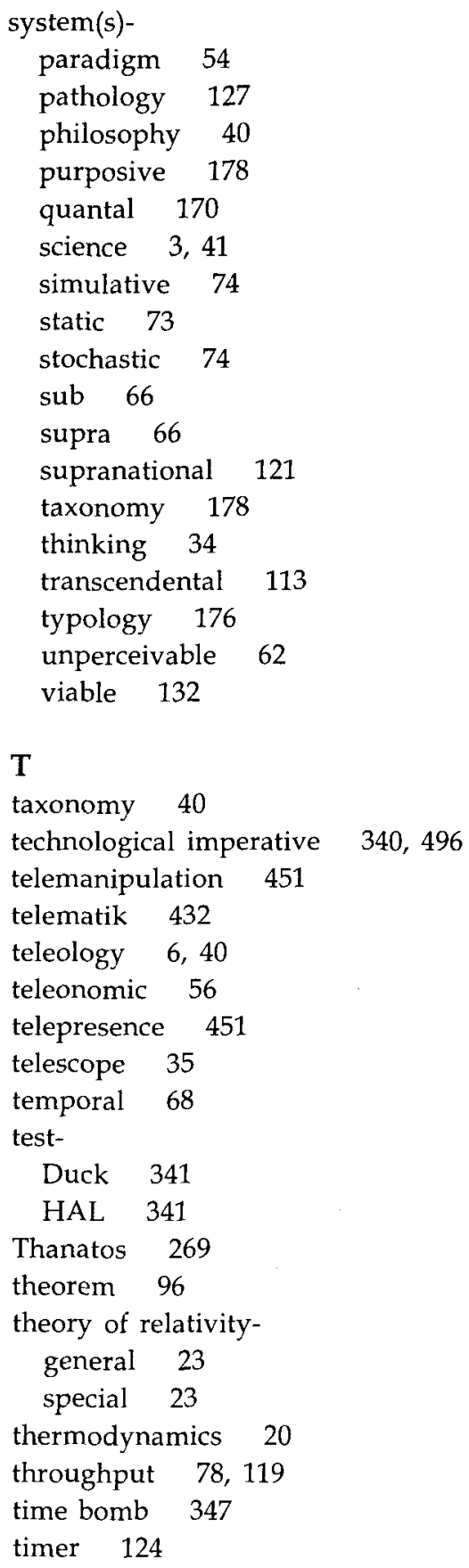




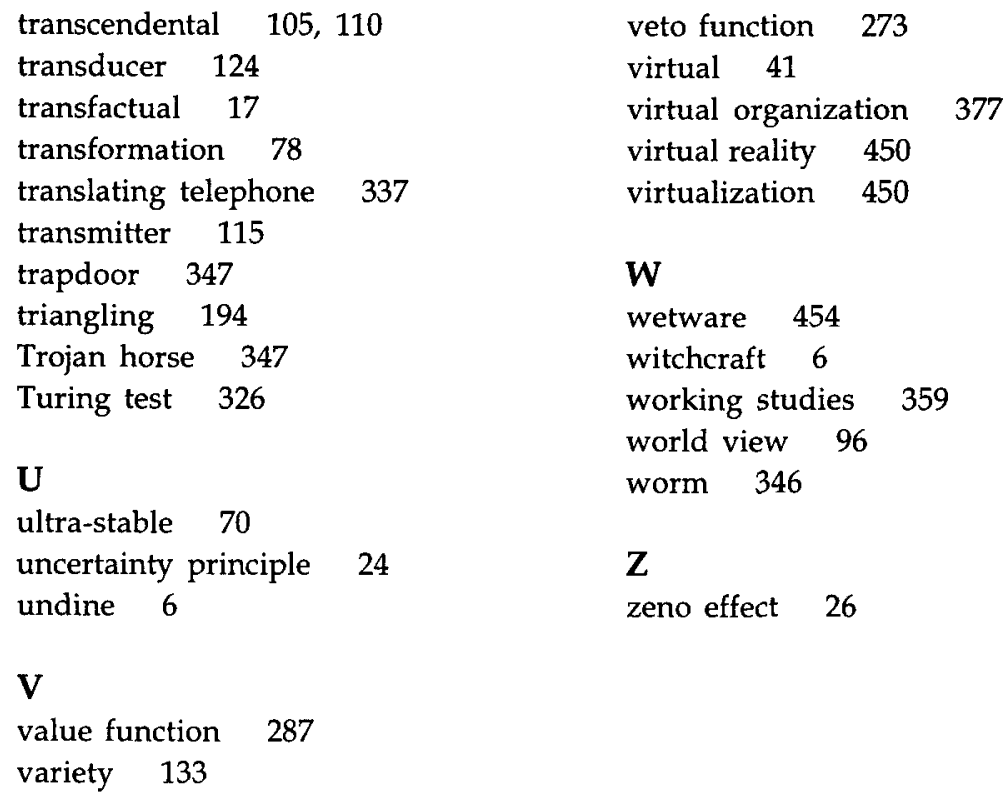

